\title{
A VISITA DOMICILIAR COMO ESTRATÉGIA DE CUIDADO NA ATENÇÃO PRIMÁRIA À SAÚDE: RELATO DE EXPERIÊNCIA
}

\section{Marcela Lages dos Santos ${ }^{1}$;Dienifer Alexsandra da Rosa Batista²; Sabrina Regina Fiorelli ${ }^{3}$;Cristiane Wagner ${ }^{4}$}

\section{RESUMO}

O presente trabalho apresenta um breve relato de experiência referente a utilização das Visitas Domiciliares (VD) como estratégia de cuidado em saúde mental, sendo esta realizada em uma Estratégia Saúde da Família (ESF) de um município do interior do Estado do Rio Grande do Sul. Tais atividades fazem parte da rotina de intervenções e estão sendo realizadas desde junho de 2021. Em relação aos métodos utilizados durante as visitas domiciliares é importante ressaltar que todas as intervenções são fundamentadas na Politica Nacional de Humanização - PNH e seus princípios e diretrizes, destacando a clínica ampliada e compartilhada que contribui na abordagem clínica do adoecimento e do sofrimento, considerando a singularidade dos sujeitos e seus diferentes saberes, buscando sempre valorizar as relações e reforçar o protagonismo do usuário frente às vivências relacionadas ao processo saúde e doença.

Palavras-chave: Estratégia Saúde da Família; Saúde Mental; Terapia Ocupacional.

Eixo Temático: Atenção Integral e Promoção à Saúde.

\section{INTRODUÇÃO}

A Atenção Primária à Saúde (APS) ou Atenção Básica (AB) tem como um de seus princípios facilitar o primeiro acesso das pessoas ao sistema de saúde, inclusive daquelas pessoas que necessitam de um cuidado em saúde mental.Segundo Szwarcwald et al (2006) a Atenção Básica apresenta-se como

\footnotetext{
${ }^{1}$ Terapeuta Ocupacional, Residente no Programa de Residência Multiprofissional em Saúde Mental da Universidade Franciscana (UFN). marcelaslages@gmail.com

${ }^{2}$ Psicóloga, Residente no Programa de Residência Multiprofissional em Saúde Mental da Universidade Franciscana (UFN). Dienifer.batista@ufn.edu.br

${ }^{3}$ Assistente Social, Residente no Programa de Residência Multiprofissional em Saúde Mental da Universidade Franciscana (UFN). fiiorelli@hotmail.com

${ }^{4}$ Terapeuta Ocupacional, Docente e Tutora de Núcleo em Terapia Ocupacional no Programa de Residência Multiprofissional em Saúde Mental da UFN. cristinewagner@gmail.com.
} 
elemento-chave para a promoção da equidade nas sociedades que apresentam grandes disparidades sociais. Sendo assim desde a sua criação em 1994 a Estratégia Saúde da Família - ESF vem cada vez mais realçando sua importância e se consolidando enquanto estratégia para a reorganização da $A B$.

A ESF conhecida anteriormente como PSF- Programa Saúde da Família teve seu início na região Nordeste do Brasil no ano de 1994, seu surgimento veio logo após a implantação do Programade Agentes Comunitários de Saúde (PACS) na década de 1990, o qual tinha como objetivo expandir e aprimorar o acesso aos serviços de saúde, utilizando como porta de entrada a atenção básica à saúde. $\mathrm{O}$ PACS é criado pelo Ministério da Saúde com o propósito de ampliar as ações da rede básica, tendo como alvo as demandas individuais e grupais, com foco no cuidado integrado e contínuo. Desse modo o PSF da origem à ESF, trazendo como principal objetivo a reafirmaçãodos princípios do SUS, e a busca pela mudança do modelo tradicional de saúde, voltado à prática clínica centrada na doença, ou seja, a ESF surge como uma possibilidade para a reorganização do modelo assistencial vigente dos serviços de saúde da época, os quais tinham em sua prática a predominância do modelo hospitalocêntrico e curativo (BRASIL,2000).

Vanderlei e Almeida (2007) apontam como principal mudança em relação à implantação da proposta da ESF "o foco de atenção, que deixa de ser centrado exclusivamente no indivíduo e na doença, passando também para o coletivo, sendo a família e seu contexto o espaço privilegiado de atuação". Posto isto, é possível afirmar que a ESF é uma estratégia pensada e elaborada para promover mudanças no atual modelo de assistência à saúde do país, permitindo que de fato sejam colocados em práticas os princípios que orientam e conduzem o Sistema Único de Saúde - SUS, os quais são: integralidade da assistência, universalidade, equidade, participação e controle social, intersetorialidade, resolutividade, saúde como direito e humanização do atendimento. Além de ainda promover o estabelecimento de vínculos e a criação de laços de compromisso e de corresponsabilização entre os profissionais e usuários dos serviços.

Desse modo, o objetivo deste trabalho é relatar a experiência da atuação em saúde mental na atenção básica utilizando como ferramenta a visita domiciliar. 


\subsection{VISITA DOMICILIAR NO CUIDADO EM SAÚDE MENTAL}

A ESF caracteriza-se comoum instrumento criado e direcionado para atender o indivíduo e a família de modo integral e constante. Para isso, se faz necessário que os profissionais desenvolvam ações de promoção,proteção e recuperação da saúde. Como já mencionado a ESF apresenta como objetivo principala reorganizaçãoda prática assistencialcom foco na família e em seu contexto físico e social.Sendo assim uma das atividades caracterizantes à ESF é a visitadomiciliar, que oportuniza ao profissional de saúde entrar no contexto familiar do sujeito e, assim, reconhecer suas demandase potencialidades(BRASIL,2000).

Segundo Mazza (1994):

A visita domiciliária constitui uma atividade de assistência à saúde exercida junto ao indivíduo, à família e à comunidade. Seu objetivo é contribuir para a efetivação das premissas de promoção de saúde definidas pela Organização Mundial de Saúde, e adoradas também, pelo Sistema Único de Saúde no Brasil. (MAZZA,1994,p.59-60).

A visita domiciliarproporciona ao profissional uma ampla visão das condições reais de vida dafamília, além de incentivar a interação familiare social do sujeito, através do conhecimento do cotidiano, dacultura, dos costumes e das crenças de umadeterminada sociedade, o que acaba por tornar essas vivênciasenriquecedoras tanto para o profissional quanto para o sujeito e sua família. É importante ressaltar que as intervenções são fundamentadas na Politica Nacional de Humanização $\mathrm{PNH}$ e em seus princípios e diretrizes, destacando a clínica ampliada e compartilhada que contribui na abordagem clínica do adoecimento e do sofrimento, considerando a singularidade dos sujeitos e seus diferentes saberes, buscando sempre valorizar as relações e reforçar o protagonismo do usuário frente às vivências relacionadas ao processo saúde e doença (BRASIL,2015).

Segundo Coelho (2004), a visita domiciliar pode ser realizada de duas formas, sendo a primeira nominada visita domiciliar Fim, a qual se apresenta com foco na atuação da atençãodomiciliar terapêutica e a visita a pacientes acamados.E a segunda chamada de visita domiciliar Meio, na qual se faz a práticada busca ativa 


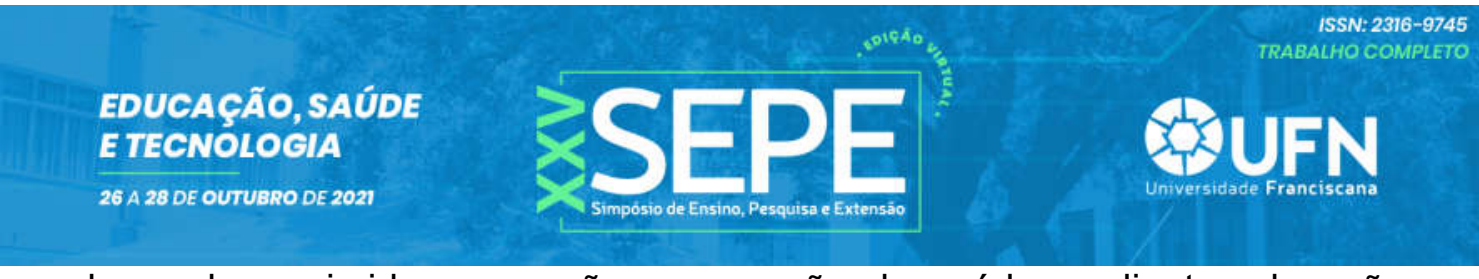

por demanda reprimida, promoção eprevenção da saúde mediante educação em saúde demaneira singularizada.

Com base na experiência vivenciada no território é possível identificar situações/casos que compreendem as duas categorias de visitas mencionadas no parágrafo anterior, porém ao fazer uma rápida análise dos casos que estão sendo acompanhados por meio de VD é perceptível que a prática aconteça em maior número nos casos em que o paciente apresenta dificuldades de locomoção ou nos casos de pacientes acamados.

\subsubsection{Terapia Ocupacional na Atenção Básica}

A Terapia Ocupacional pode ser definida como:

A Terapia Ocupacional é um campo de conhecimento e intervenção em saúde, em educação e na área social, que reúne tecnologias orientadas para a emancipação e autonomia de pessoas que, devido a problemáticas específicas (físicas, sensoriais, psicológicas, mentais ou sociais), apresentam dificuldades de inserção e participação na vida social temporária ou definitivamente. (BARROS E COL.,2002, p.366).

A Terapia Ocupacional vem de um contexto histórico centradono modelo biomédico com ênfase na reabilitação física, sendo uma área que por muito tempo teve sua atuação subordinada e limitada pela medicina. Hoje já é possível ver o profissional terapeutaocupacional atuando em vários cenários e contextos com diferentes abordagens.Porém sua atuação na Atenção Primária/Atenção Básica ainda tem suas limitações.

Apesarde ser um profissional de extrema importância como provedor de saúde, podendo atuar dediferentes formas na construção coletiva com a equipe, nos grupos, no atendimentoindividualizado e na comunidade, principalmente no contexto da ESF a qual possui comoprincipal estratégia as visitas domiciliares, onde o profissional pode identificar a realdemanda daquela família, daquele usuário e da própria comunidade em si, mesmo assim sua atuação na $A B$ apesar dos avanços ao longo dos anos ainda pode ser considerada restrita.

O terapeuta ocupacional por não fazer parte da equipe mínima, vê sua atuação na $A B$ delimitada, sendo reconhecida nesse contexto após a criação dos 
EDUCAÇÃO, SAÚDE

ETECNOLOGIA

26 A 28 DE OUTUBRO DE 2021

QUFN

Núcleos de Apoio à Saúde da Família (NASF) por meio da Portaria GM n 154 dejaneiro de 2008. O NASF é constituído por equipes compostas por profissionais de diferentes áreas de conhecimento, inclusive a terapia ocupacional, para atuar em conjunto com os profissionais das equipes de saúde da família, visando ampliar o escopo e a resolutividade de atenção aos usuários (BRASIL, 2008).

\section{METODOLOGIA}

Trata-se de um relato de experiência referente a utilização da visita domiciliar como ferramenta potente no cuidado em saúde mental, com enfase na atuação da terapia ocupacional nesse contexto. A atuação acontece por meio do Programade Residência Multiprofissional em Saúde Mental da Universidade Franciscana (UFN), realizada em uma Estratégia Saúde da Família (ESF) de um município do interior do Estado do Rio Grande do Sul. Tais atividades fazem parte da rotina de intervençõese estão sendo realizadas desde junho de 2021.Sendo que, a frequência, a duração e o tipo de ações propostas durante as visitasdomiciliares foram definidas de acordo com as subjetividades, necessidades e singularidadesdos usuários e familiares que estão em acompanhamento.

\section{RESULTADOS E DISCUSSÕES}

O processo de trabalho em saúde mental na referida ESF acontece por meio do programa de Residência Multiprofissional em Saúde Mental da Universidade Franciscana. A equipe multiprofissional de saúde mental é composta por uma terapeuta ocupacional, uma psicológa, uma assistente social e uma farmacêutica.

Desde os anos setenta e com base em todo o processo da reformapsiquiátrica os conceitos e práticas relacionados àatenção a saúde mental vêm sofrendo transformações ao longo dos anos. Desde seu início a reforma psiquiátrica apresentava e ainda apresenta como eixo centralizador a desinstitucionalização e a redução donúmero de leitos nos manicômios, além da implementação da rede comunitária de serviços substitutivos (TANAKA E RIBEIRO,2009).

Segundo Tanaka e Ribeiro (2009): 
(...) este direcionamento da reforma psiquiátrica para o cuidado dos pacientes com transtornos severos e persistentes e para a implantação deCentros de Atenção Psicossocial (CAPS) deixou emsegundo plano a assistência aos transtornos mentais menos graves e mais prevalentes. (TANAKA E RIBEIRO,2009, p.478-479).

E é na assistência aos transtornos mentais menos graves e mais prevalentes que a ESF se destaca, por ter no centro das suas práticas o trabalho multiprofissional realizadoem um determinado território adstrito onde desenvolvemse ações de saúde, baseadas no conhecimento da realidade local e das necessidades observadas. Ter acesso ao contexto familiar e social do paciente proporciona ao profissional um olhar mais detalhado as reais demandas, além de proporcionar uma melhor vinculação para com o paciente, tornado o cuidado mais integral e eficaz.

Tendo em vista a problemática levantada pelos autores referente a falta de cuidados para com os casos considerados menos graves, a Coordenação Geral da Saúde Mental(CGSM) no ano de 2001 desenvolveu uma sucessão de documentos referentes a articulação entre a saúde mental e a atenção básica, documentos esses que apresentam como principais diretrizes o apoio matricial; a priorização da saúde mental na formação profissional dasequipes da $A B$ e intervenções de acompanhamento e avaliação dasações de saúde mental desenvolvidas na atenção básica (TANAKA E RIBEIRO, 2009).

A Organização Mundial de Saúde (1990), publicou o documento "La introducción de un componente de salud mental en la atención primaria", no qual ressalta a magnitude do componente emocional na atenção à saúde: "É impossível alcançar saúde se não se cuidar das necessidades emocionais" (ORGANIZAÇÃO MUNDIAL DE SAÚDE,1990, p. 60).

Diante do exposto é irrefutável a relevância e a importância da AB no cuidado em saúde mental. Segundo Silveira e Vieira (2009):

A ESF estabelece uma forte interface com princípios caros à reforma psiquiátrica brasileira, como as noções de territorialidade e responsabilização pela demanda, além de conferir um novo sentido e ordenamento às ações de saúde mental no contexto da atenção básica, tornando possível migrar do modelo das psicoterapias tradicionais para um modelo onde o usuário seja considerado como sujeito-social, numa 

suas redes sociais e ambiente ecológico (SILVEIRA E VIEIRA,2009, p.140141).

Sendo a ESF uma importante estratégia de organização e cuidado em saúde mental é imprescindível ressaltar a visita domiciliar como ferramenta potente no cuidado do indivíduo em seu contexto de produção de vida, onde a terapêutica se estabelece com o olhar para além da "doença", direcionando-se para os sujeitos, suas subjetividades e seu cotidiano, através da aproximação da realidade do sujeito e a criação de vínculos.

A atuação na ESF tem acontecido por meio de visitas domciliares e atendimentos individuais, os grupos ainda não estão acontecendo devido ao cenário pandêmico. Esses atendimentos normalmente são realizados via encaminhamento realizado pelo médico da saúde da família da unidade, sendo necessário o paciente passar por uma consulta e avalição, para posterior encaminhamento ao atendimento em saúde mental. Os atendimentos são realizados de forma conjunta, com no mínimo dois núcleos diferentes e nos casos mais complexos os atendimentos são realizados de forma conjunta com os quatro núcleos. Essa atuação multiprofissional apresenta muitos benefícios e ganhos para o paciente e sua família, que são acolhidos nesse processo saúde-doença de forma integral, contando com diferentes visões em relação ao tratamento e aos cuidados, já o profissional que está inserido em uma equipe multiprofissional desfruta da troca de saberes com os diferentes núcleos, ampliando sua visão frente ao cuidado em saúde e atuando de forma mais integral e continuada.

Posto isto, ressalto a atuação do terapeuta ocupacional junto a equipe multiprofissional e ao pacienteno seu contexto domiciliar. O terapeuta ocupacional compreende projetos, realizações, sentido e resgate de atividades significativas, além da busca pela autonomia, auxiliando no processo de restabelecimento e melhoria da qualidade de vida por meio da horizontalidade e integralidade das relações. Apesar da VD ser algo relativamente novo em nossa prática, pois começamos a utilizar essa ferramenta apenas em junho deste ano, já é possível observar alguns dos benefícios que está ferramenta proporciona no cuidado integral para com o paciente e sua família. 
Atualmente estamos realizando o acompanhamento por meio de VD de sete pacientes, todos apresentam dificuldades de ordem física o que acaba dificultando o seu acesso a unidade, sendo que a frequência, a duração e o tipo de ações propostas durante as visitas domiciliares são definidas em conjunto com a equipe e o paciente, respeitando sempre suas subjetividades, necessidades e singularidades.

\section{CONCLUSÃO}

O desenrolar deste estudo possibilitouuma melhor compreensão sobre a importância das visitas domiciliares no território, mostrando que a mesma apresenta pontospositivos por fornecer assistência a uma parte dapopulação que normalmente não teria acesso aosserviços de saúde, seja por questões de ordem física como mencionado anteriormente no texto, como os acamados ou pessoas com alguma limitação física ou em decorrência de alguma outra questão que impossibilite ou dificulte o acesso a unidade de saúde. A visita domiciliar utilizada como principal ferramenta de cuidado dentro do território através da ESF nos possibilitou fazer uma reflexão sobre a utilização desta prática no cuidado em saúde, dentro do contexto dos pacientes, evidenciando ser uma importante ferramenta no cuidado integral e familiar, ferramenta esta que permite um cuidado mais humanizado, com maior troca de informações e melhor criação de vínculo,desta forma a VD contribui para uma construção e corresponsabilização coletiva referente ao cuidado em saúde.Este trabalho buscou expor por meio de um breve relato de experiência o potencial da visitadomiciliar como elemento de aproximação das equipes desaúde com as famílias. Essa prática permite a construção de vínculos e um atendimento mais humanizado, visando a prevenção e promoção de saúde e melhoria na qualidade de vida das famílias atendidas.

\section{REFERÊNCIAS}

BRASIL. Portaria GM n 154, de 24 de janeiro de 2008. Cria os núcleos de apoio à saúde da família. Brasília: Diário Oficial da República Federativa do Brasil [2008]. Disponível 
Acesso em: 22 set.2021.

BRASIL. Ministério da Saúde (MS). Secretaria de Políticas de Saúde-Departamento de Atenção Básica. Caderno de Atenção Básica. Programa Saúde da Família: a implantação da Unidade de Saúde da Família.Brasília: Editora do Ministério da Saúde; 2000. (1 $1^{\text {a }}$ edição ).

BRASIL. Ministério da Saúde (MS). Política Nacional de Humanização. Humaniza SUS.Brasília: Ministério da Saúde; 2015. (1ª edição $2^{\mathrm{a}}$ reimpressão).

COELHO, F.L.G, SAVASSI, L.C.M. Aplicação da escala de risco domiciliares.Revista Brasileira de Medicina de Família e Comunidade. Minas Gerais, v. 1 n. 2, p. 19-26,2004.

MAZZA, M.M.P.R. A visita domiciliária como instrumento de assistência de saúde. Revista Brasileira de Crescimento e Dessenvolvimento Humano. São Paulo, v. 4 n.2, p.60-68, 1994.

ORGANIZAÇÃO MUNDIAL DE SAÚDE.A introdução de um componente de saúde mental na atenção primária. Genebra: Organização Mundial da Saúde; 1990. 60 p. Título original: La introducción de un componente de salud mental en la atención primária. ISBN 92-4-356136-7.

BARROS, D.D, LOPES, R.E., GALHEIGO, S.M,Projeto metuia - Terapia Ocupacional no campo social.O Mundo da Saúde, São Paulo:v.26, n.3, p.365-369, 2002.

SZWARCWALD C.L, MENDONÇA M.H.M, ANDRADE C.L.T. Indicadores de atenção básica em quatro municípiosdo Estado do Rio de Janeiro, 2005: 


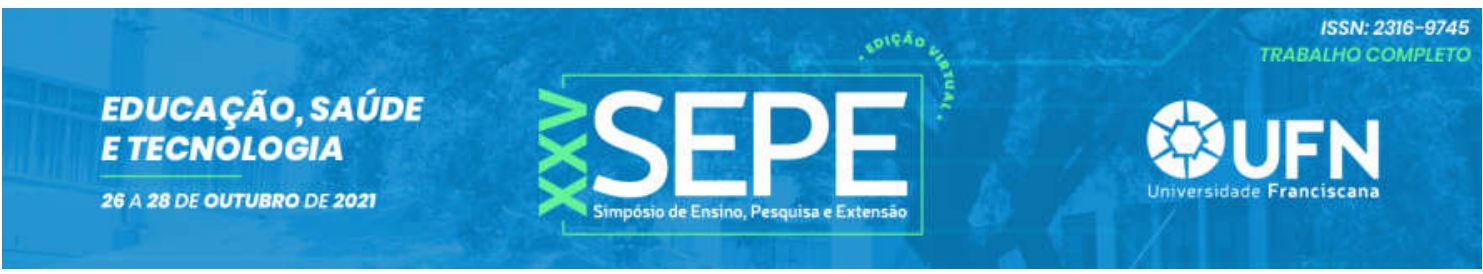

resultados deinquérito domiciliar de base populacional. Ciência \& Saúde Coletiva. São Paulo, v.11 n.3, p.643-655, 2006.

SILVEIRA, D.P, VIEIRA, A.L.S. Saúde mental e atenção básica em saúde:análise de uma experiência no nível local. Ciência \& Saúde Coletiva. São Paulo, v.14 n.1, p.139-148,2009.

TANAKA O.Y, RIBEIRO E.L. Ações de saúde mental na atenção báscia: caminho para ampliação da integralidade da atenção. Ciência \& Saúde Coletiva. São Paulo, v.14 n.2, p.477-486, 2009.

VANDERLEI MIG, ALMEIDA MCP. A concepção e prática dos gestores e gerentes da estratégia de saúde dafamília. Ciência \& Saúde Coletiva. São Paulo, v.12 n.2, p.443-453, 2007. 